\title{
Wem gehört die Stadt?
}

\section{Eigentum in der Debatte um bezahlbares Wohnen}

\section{Whose city is it, anyway? \\ Property and the debate on affordable housing}

\section{Niklas Angebauer, Oldenburg \& Tobias Habermann, Dortmund}

Zusammenfassung: Der Beitrag systematisiert, kontextualisiert und evaluiert den eigentumstheoretischen Hintergrund der derzeit breit geführten Debatte um bezahlbaren Wohnraum in deutschen Großstädten. Ausgehend von der These, dass hinter der sogenannten ,Wohnungskrise“ eigentlich eine ,Bodenkrise ${ }^{6}$ steht, werden drei zentrale Konflikte um die politische Rahmung des Eigentums an Boden diskutiert: die Frage nach dem Gebrauch (Wer entscheidet darüber, welchem Zweck der Boden zugeführt wird?), der Verwertung (Wer profitiert von Bodenwertsteigerungen?) und dem Eigentumsschutz (Wer kann sich legitimerweise auf den Eigentumsschutz berufen?). Dabei werden die normativen Potentiale eines immanenztheoretischen, apriorisch pflichtgebundenen Eigentumsverständnisses für eine zeitgemäße Wohnraum- und Bodenpolitik ausgelotet.

Schlagwörter: Eigentum, Bodenpolitik, Stadtentwicklung, Freiheit, Leistungsbezug

Abstract: The article systematizes, contextualizes and evaluates the property theoretical foundation of the ongoing debate on affordable housing in German major cities. Starting from the argument that the so-called 'housing crisis' is a 'land crisis' at its core, we discuss three crucial issues linked to the political framing of land property: rights of use (who decides about the land use on a building plot?), rights of profit (who benefits from land value increases?), and property as a subjective right (who should be entitled to full property rights?). The paper thereby fathoms the normative potentials of a conception of property to which obligations are immanent as a sound groundwork for contemporary housing and land policy. 
Keywords: Property, land policy, urban development, freedom, labour.

\section{Einleitung: Wohnungskrise, Bodenkrise und Eigentumspolitik}

„Weniger Sozialismus, mehr Wohneigentum“, ruft der FDP-Abgeordnete Dr. Florian Toncar in den Saal des Bundestags (Deutscher Bundestag 2019a, 17099). In der Plenarsitzung herrscht Einigkeit über das Ziel: Alle Fraktionen wollen die Entlastung der Wohnungsmärkte. Aber müssen dafür Spekulanten abgeschreckt werden (Caren Lay, DIE LINKE)? Oder wäre das ein „Motivationskiller“ für dringend benötigte Investoren, die Geld zur Linderung der Wohnungsnot in die Hand nehmen (Alexander Hoffmann, CDU)?

Während die Politik über die Lösung der „sozialen Frage unserer Zeit“ (Horst Seehofer) streitet, wird die Luft auf den Wohnungsmärkten in Deutschland von Jahr zu Jahr dünner. ${ }^{1}$ Dass während des Lockdowns Menschen in überbelegten Wohnungen regelrecht „eingepfercht“ waren (um den epidemiologischen Fachausdruck zu verwenden; vgl. RKI 2015), ist ein weiteres plakatives Beispiel dieser Entwicklung: Angemessener Wohnraum ist ein Privileg, das sich immer weniger Menschen leisten können. Mieter*innen und Medien lenken ihre Aufmerksamkeit auf die steigenden Mieten, schauen damit aber bloß auf das Ende der Kausalkette, denn steigende Mieten sind nicht die Ursache der Knappheit, sondern deren Folge (Löhr 2018a). Veränderungen des Mietrechts stellen allenfalls eine „Notbremse“ dar (Prantl 2018, 2), lassen aber das zugrundeliegende Problem unberührt, welches buchstäblich - tiefer liegt: Wer die Wohnungsnot verstehen möchte, muss nicht primär Mieten oder Gebäude in den Blick nehmen, sondern Grund und Boden selbst.

Was ein solcher Blick zutage bringt, ist ernüchternd: Die geringe Verfügbarkeit und das hohe Preisniveau von Grund und Boden lassen einen

1 Die Angebotsmieten verzeichnen in strukturstarken Großstädten einen Anstieg von fünf bis sieben Prozent pro Jahr (Deutscher Bundestag 2019b), was Haushalte mit geringem Nettoeinkommen überdurchschnittlich hart trifft (Lebuhn und Holm 2017). Der Trend schwächte sich mancherorts pandemiebedingt ab, zugleich erleiden Haushalte aufgrund von Kurzzeitarbeit und Stellenabbau finanzielle Einbußen (Statistisches Bundesamt 2020; BBSR 2021). In deutschen Großstädten fehlen bis zu 1,9 Millionen Wohnungen für einkommensschwache Haushalte (Holm et al. 2018); auch die soziale Segregation nimmt zu (Helbig und Jähnen 2018). 
privaten Wohnungsbau zu günstigen Mieten vielfach nicht mehr zu (Waltersbacher 2017). Auf den Bodenmärkten deutscher Großstädte ist seit 2009 ein sprunghafter Anstieg der Preise zu verzeichnen, während das Angebot an Bauland weitgehend stagniert (AKOGA 2019, 161). Im Bundesdurchschnitt sind Neubauprojekte erst ab einer Erstvermietungsmiete von zehn bis zwölf Euro pro Quadratmeter (nettokalt) rentabel (Waltersbacher 2017, 15) und in den innerstädtischen Lagen von Berlin lässt der Verwertungsdruck fast ausschließlich den Neubau von Eigentumswohnungen zu (SenStadt Berlin 2019, 31). Dies ist mitnichten ein tagesaktuelles oder deutsches Phänomen: In den Industrieländern des Globalen Nordens sind 8o Prozent des Anstiegs von Immobilienpreisen zwischen 1950 und 2012 direkt auf die Bodenpreise zurückzuführen (Knoll et al. 2017). Es reicht daher nicht aus, die Immobilienwirtschaft als alleinige Verursacherin und Nutznießerin steigender Mieten zu brandmarken: „Wer die hohen Grundstückspreise zahlt und kein finanzstarker Philanthrop ist, der muss ein Haus mit teuren Miet- und Eigentumswohnungen darauf errichten, damit er seine Kosten wieder finanziert bekommt. Es hilft nichts, das zu verteufeln, wenn man nicht das gesamte System in Frage stellt" (Weißmüller 2018, 10).

Die Wohnungskrise ist also in erster Linie eine Bodenkrise. Drei Faktoren sind hier entscheidend. Erstens die Eigenlogik des Guts ,Boden“: Boden ist seinem Wesen nach knapp, unvermehrbar und immobil, sodass einer Nachfragesteigerung nicht kurzerhand mit einer Angebotsausweitung begegnet werden kann. Zweitens ist die Nachfrage an Boden und Wohnfläche über die letzten Jahrzehnte besonders im urbanen Raum stetig gestiegen. Vordergründig sind hier demographische und konjunkturelle Triebkräfte von Bedeutung, wie der Zuzug aus dem In- und Ausland, wachsende Erwerbstätigenzahlen, sinkende Arbeitslosigkeit, steigendes Einkommen (Held und Waltersbacher 2015) und geänderte Nachfragemuster. ${ }^{2}$ Hinzu kommt, dass Niedrigzinsphasen - derzeit verstärkt durch die Geldpolitik der Europäischen Zentralbank (EZB) - Wohneigentum durch günstige Kredite erschwinglicher machen (Waltersbacher 2017). In Zeiten des „Anlagenot-

2 Die Corona-Pandemie dient als Katalysator mancher, schon zuvor evidenter Entwicklungen (Digitalisierung der Arbeitswelt, Umsatzanstieg im Online-Handel), bei denen räumliche Folgen absehbar sind (flexible Wohnortwahl, Strukturwandel der Innenstädte). Aus dem pandemiebedingten Nachfrageanstieg für das „Wohnen im Grünen“ lassen sich indes noch keine fallenden Wohnimmobilienpreise in den Zentren ableiten (Anders et al. 2020). 
stands“ (Löhr 2018b, 13) sind Investitionen in Immobilien und Liegenschaften mit vergleichsweise hohen Cash-Flow-Renditen und zu erwartenden Wertsteigerungen attraktiv, was zur fortschreitenden „Finanzialisierung der Immobilienwirtschaft“ beiträgt (Holm 2011, 12). Der dritte - und für diesen Artikel zentrale - Faktor ist die eigentumspolitische Rahmung des Boden- und Immobilienmarkts. Denn der Wert von (und die Nachfrage nach) Grundstücken hängt empfindlich davon ab, welche Gebrauchs- und Verwertungsmöglichkeiten ihren Eigentümer*innen offenstehen.

Vor diesem Hintergrund erweist sich die gegenwärtige Wohnungsbzw. Bodenkrise zumindest teilweise als Produkt einer spezifischen, vom Primat der Privatnützigkeit geprägten Eigentumspolitik. Diese wurde in den letzten Jahrzehnten kaum herausgefordert, sondern allenfalls ausgebaut: Seit den 1990er Jahren haben sich bodenrechtliche Reformbemühungen auf fragmentarische Novellen zur Beschleunigung von Planungsprozessen beschränkt, so etwa die Einführung vereinfachter Bebauungsplanverfahren im Innen- und Außenbereich oder die Gewährung finanzieller Hilfen (Baukindergeld) (Bunzel und Niemeyer 2018). Diese Maßnahmen berühren das Eigentumsgrundrecht nur insoweit, als dass sie die Handlungsspielräume privater Grundeigentümer*innen über Ausnahmen, Erleichterungen und finanzielle Unterstützung erweitern. Auch im aktuellen Koalitionsvertrag betont das Kabinett Merkel IV, keine weiteren „Verschärfungen der Eingriffsmöglichkeiten der Kommunen“ (CDU, CSU und SPD 2018, Z. 5114-5119) in das Eigentum Privater vornehmen zu wollen, und rückt damit auch rhetorisch von einer stärkeren Gemeinwohlbindung des Grundeigentums ab.

Angesichts der manifesten Wohnungskrise wird diese eigentumspolitische Zurückhaltung zunehmend erklärungsbedürftig. Denn die eigentumspolitische Rahmung des Boden- und Immobilienmarktes obliegt explizit dem Gestaltungsauftrag des Gesetzgebers: Nach Art. 14 GG hat er Inhalt und Schranken des Eigentums zu bestimmen und dabei eine sachgerechte Balance zwischen der Privatnützigkeit und der Sozialpflichtigkeit des Eigentums zu wahren. Das Problem der Wohnungsnot stellt den Gesetzgeber vor die Frage, wie stark er das Eigentum Privater sozialpflichtig einbinden muss, um das Gemeinwohlziel, d.h. die Entlastung der Bodenmärkte zu erreichen. Wird der Baulandmangel durch die rechtliche Stellung der privaten Eigentümer*innen verursacht (DIFU und vhw 2017) oder gilt es vielmehr, das Privateigentum aus den „regulativen, die Bürger einschränkenden“ Baunormen herauszulösen (Haus \& Grund 2019)? Mit anderen Worten: Wie sähe 
eine angemessene eigentumspolitische Rahmung urbaner Boden- und Wohnungsmärkte aus?

Indem wir diese Frage stellen, schließen wir an eine u. a. in der Stadtsoziologie und Humangeographie breit geführte und überaus ertragreiche Debatte darüber an, wem die Stadt „gehört“ bzw. gehören sollte (Lefebvre 2016; Harvey 2008). Während die Frage dort meist metaphorisch verstanden und unter dem Stichwort „Recht auf Stadt“ mit einer heterogenen Sammlung von politischen, ökonomischen und sozialen Zugangs- und Partizipationsrechten beantwortet wird, wollen wir die Frage aber einmal ganz beim Wort nehmen und uns der Antwort eigentumstheoretisch nähern. ${ }^{3}$

Dieses Vorgehen wird dadurch verkompliziert, dass der Begriff „Eigentum" selbst umstritten ist und nicht nur auf eine, sondern auf mehrere begriffliche Traditionslinien zurückgeführt werden kann. Indem wir eine heuristische Ordnung dieser verschiedenen Begriffstraditionen entwickeln (Abschnitt 2), schaffen wir ein Koordinatensystem, in dessen Rahmen wir aktuelle eigentums- und bodenpolitische Konflikte analysieren, verorten und kritisch bewerten können. Dabei unterscheiden wir zwischen einer absoluten und einer apriorisch pflichtgebundenen Immanenztheorie des Eigentums. Wie wir im darauffolgenden Abschnitt herausarbeiten, verweist die Frage „Wem gehört die Stadt?" auf drei Konfliktfelder des Eigentums. Erstens auf das Gebrauchsrecht (3.1): Wer entscheidet darüber, welchen Zwecken der Boden zugeführt wird?; zweitens auf das Verwertungsrecht (3.2): Wem stehen die Bodenwertsteigerungen zu?; und drittens auf die Reichweite des Eigentumsschutzes (3.3): Welche Akteur*innen können sich legitimerweise auf ihn berufen?.

In Auseinandersetzung mit diesen drei Debattensträngen zeigen wir die normativen Potenziale eines immanenztheoretischen Eigentumsbegriffs auf, dem zufolge Eigentümer*innen nicht pauschale, sondern von Anfang an qualifizierte und pflichtgebundene Rechte zustehen, die je nach Eigentumsobjekt und -subjekt variieren. Dieses auf den ersten Blick bescheidene Ergebnis ermöglicht, so unser Argument, zugleich eine effektive Gemeinwohlbindung der Bodennutzung und eine nach Akteurstyp differenzierte Reichweite des Eigentumsschutzes.

$3 \quad$ Neben der hier gewählten rechtstheoretisch-normativen Perspektive gibt es zahlreiche weitere Ansätze, die zur Debatte um bezahlbaren Wohnraum Erhellendes beizutragen haben. Dazu gehören neben den im Text Genannten u. a. Sennett (2018) (Soziologie), Bauman (2018) und Rötzer (2020) (Philosophie) sowie Hubeli (2019) und Hoffmann-Axthelm (1993) (Städtebau). 


\section{Zum Begriff des Eigentums}

In erster Annäherung bezeichnet Eigentum das subjektive Recht, exklusiv über eine Sache zu verfügen. In dieser formalen Bestimmung liegt der kleinste gemeinsame Nenner, der allen Eigentumstheorien gemein ist: Eigentumsrechte sind subjektive Rechte, die ihre Täger*innen dazu bemächtigen, unbefugte Dritte von einer Sache auszuschließen (Exklusionsrecht) und frei über die Sache zu verfügen, sie also selbstbestimmten Zwecken zuzuführen (Verfügungsrecht). Dieses exklusive Verfügungsrecht hat zwei Dimensionen: Einerseits kann es sich auf den Gebrauch der Sache richten (was das Recht auf Nutzung, Verbrauch, Veränderung, Steuerung und Management beinhaltet); andererseits auf deren Verwertung (als Recht auf Aneignung der Erträge, die aus der Sache generiert werden). Ein solch formaler Eigentumsbegriff ist nicht auf das private Eigentum Einzelner verengt - Eigentum in diesem Sinne kann ebenso gut von Kollektiven oder der öffentlichen Hand besessen werden. 4

Jenseits dieser formalen Bestimmung aber beginnen die Kontroversen. Gestritten wird in der Eigentumstheorie insbesondere um die Reichweite des Eigentumsrechts: Welche Befugnisse kommen Dritten zu? Und was gilt als ,selbstständige ' Verfügung bzw. wie umfassend müssen die Verfügungsbefugnisse von Eigentümer*innen gedacht werden? Während die absolute Theorie das Eigentum als zunächst unbeschränkt konzipiert (2.1), sehen Immanenztheorien es als konstitutiv beschränkt an (2.2). Diese heuristische Typologie der Eigentumstheorien bietet uns die Grundlage, um wohnungs- und bodenpolitische Debatten zu kontextualisieren (s. Abb. 1).

\subsection{Absolute Theorie}

Für die absolute Theorie des Eigentums stellt das unbeschränkte Privateigentum das Paradigma dar, von dem sich alle anderen Eigentumsformen (beschränktes, kollektives oder öffentliches Eigentum) ableiten. Paradigmatisch für diese Konzeption steht die berühmt gewordene Formulierung des englischen Juristen William Blackstone, das Eigentum bezeichne die „despotische Herrschaft [...] über die Dinge der äußeren Welt unter vollkommenem Ausschluss der Rechte aller anderen Individuen des Universums“ (Blackstone 2016, II, 1). Dieses Recht gilt zunächst für alle Eigentumsobjekte gleichermaßen - egal, ob es sich um die eigene Krawatte, eine Krawattenfabrik, 


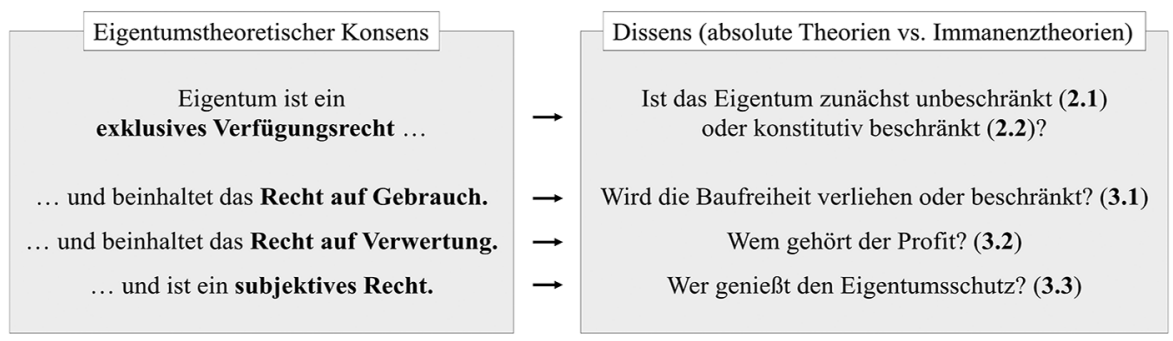

\section{Abbildung 1}

den Vorgarten oder eine landwirtschaftliche Nutzfläche handelt. ${ }^{5}$ Wohlgemerkt, diese Einheitlichkeit des Eigentums bezieht sich auf die innere Logik des Eigentumsbegriffs, nicht auf die tatsächliche Rechtsstellung von Eigentümer*innen. Letztere bleibt i.d.R. „von außen“ durch öffentlich-rechtliche Gesetze beschränkt. ${ }^{6}$

Wesensmerkmal des Eigentums ist demnach die (zunächst) unbeschränkte Exklusions- und Verfügungsbefugnis. Die primäre Legitimationsgrundlage des so verstandenen Eigentums ist ein negativer Freiheitsbegriff, der individuelle Freiheit als Abwesenheit von äußerem, insbesondere staatlichem Zwang deutet. Dem liegt die Vorstellung zugrunde, dass die Freiheit der Bürger*innen der bürgerlichen Gesellschaft vorausgeht und insbesondere vor dem hoheitlichen Zugriff des Staates geschützt werden muss. Das Eigentum gibt diesem Schutz eine institutionelle Form: Es versetzt Bürger*innen in die Lage, dem Staat gestärkt, selbstbewusst und auch unbequem gegenüberzutreten (Papier und Shirvani 2019, Art. 14, Rn. 2). Während dieser jeden Eingriff in das Eigentum durch Gemeinwohlgründe rechtfertigen

$5 \quad$ Es bleibt umstritten, wie weit die Quellen des absoluten Eigentumsbegriffs zurückreichen; diskutiert werden Vorläufer und Weichenstellungen im römischen Recht (Garnsey 2007), dem christlichen Armutsstreit oder der Eigentumslehre des Bartolus (Schermaier 2017; Tierney 1997). Klar artikuliert und institutionalisiert wird der absolute Eigentumsbegriff aber erst im Zuge der bürgerlichen Revolutionen der Moderne: Art. 544 des Code Civil bspw. definiert das Eigentum als „unbeschränktes Recht zur Nutzung und zur Verfügung über die Dinge“ (le droit de jouir et disposer des choses de la manière la plus absolue).

6 Zudem entscheiden Eigentümer*innen selbst, ob sie von ihrem Ausschlussrecht Gebrauch machen. Insofern gleicht Eigentum weniger einer Mauer als vielmehr einem Tor, durch das eine selektive Teilhabe ermöglicht werden kann (vgl. Penner 1996, 744; siehe dazu Davy 2018). 
muss, ist die Verfügungsfreiheit der Eigentümer*innen zunächst von jeder Rechenschaftspflicht befreit (Depenheuer und Froese 2018, Art. 14, Rn. 29). Der Gesetzgeber kann zwar individuelle Freiheit durch öffentliches Recht beschränken, aber er hat keine „unumschränkte Macht, positiv zu bestimmen, was Freiheit ist“ (ebd., Rn. 41). Die Möglichkeit, dass der Staat gerade durch die öffentlich-rechtliche Beschränkung privater Verfügungsmacht Freiheit realisieren oder schützen könnte, ist so schon aus begrifflichen Gründen ausgeschlossen.

Auffällig ist, dass die absolute Theorie des Eigentums nicht nur von Konservativen oder Wirtschaftsliberalen damals wie heute regen Zuspruch erhält, ${ }^{7}$ sondern ebenso von vielen der schärfsten Kritiker*innen des Eigentums vertreten wird. Eine besonders im (Post-)Marxismus, aber auch in der feministischen oder ökologischen Eigentumskritik weitverbreitete Strategie übernimmt den absoluten Eigentumsbegriff, versucht aber zu zeigen, dass dieser als ganzer unhaltbar (und die Eigentumsidee somit insgesamt abzulehnen) ist. ${ }^{8}$ Diese Strategie einer totalisierenden Eigentumskritik erlangt ihre moderne Form im Frühwerk Pierre-Joseph Proudhons. Ebenso wie die bürgerlichen Juristen seiner Zeit projiziert Proudhon den absoluten Eigentumsbegriff des Code Civil auf die römische Antike zurück, aber nicht, um dem Eigentum Autorität zu verleihen, sondern, im Gegenteil, um argumentieren zu können, dass das Eigentum notwendig das Recht auf den willkürlichen Missbrauch der Sache einschließe und daher insgesamt abzulehnen (oder zumindest moralisch ambivalent zu bewerten) sei. ${ }^{9}$ Eine solche Kritik akzeptiert die begriffliche Engführung des Eigentums auf einen negativen Freiheitsbegriff, sucht aber zu zeigen, dass dieser in letzter Konsequenz selbstwidersprüchlich wird, weil er zu einer Bedrohung für die gleiche Freiheit aller wird. An diese Strategie knüpfen - wenn auch oft in differenzierterer und historisch versierterer Form - moderne Vertreter*innen

7 Vgl. exemplarisch Depenheuer und Froese (2018, Rn. 47): „Eigentum in diesem Sinne ist also grundsätzlich ein verfassungsrechtlich freies, unbeschränktes und absolutes Recht.“

8 Vgl. exemplarisch Hardt und Negri (2010), Sutterlütti und Meretz (2018) sowie Loick (2016).

9 „Es steht dem Eigentümer frei, seine Früchte noch an den Ästen verfaulen zu lassen, Salz auf sein Feld zu säen, seine Kühe über dem bloßen Boden zu melken, einen Weinberg in eine Wüste zu verwandeln oder einen Park aus einem Gemüsegarten zu machen" (Proudhon 2018, 54). 
einer totalisierenden Eigentumskritik an, wenn sie den begrifflichen Kern des (Privat)Eigentums in einer „Freiheit der Beschädigung“ (Nuss 2019, 99) sehen oder darauf zielen, „grundsätzlich jede eigentumsförmige Beziehung zwischen Menschen und Welt zu kritisieren“ (Loick 2016, 10).

\subsection{Immanenztheorien}

Immanenztheorien wenden sich sowohl gegen affirmative als auch gegen kritische absolute Theorien des Eigentums. Sie machen geltend, dass ein wohlverstandener Eigentumsbegriff nicht unbeschränkt sein kann, sondern vielmehr konstitutiven Schranken unterliegt: Die Eigentumsgrenzen sind dem Eigentum immanent (vgl. Wesche 2014). Als ein paradigmatischer Vertreter der Immanenztheorie gilt etwa John Stuart Mill, der argumentiert, die Eigentumsgesetze hätten sich „noch nirgends den Prinzipien angepasst, auf denen die Rechtfertigung des Privateigentums beruht“ (Mill 2016, 234). Als legitimes Eigentum im eigentlichen Sinne könne aber nur gelten, was diesen Prinzipien auch entspreche. Anstatt dem Phantasma einer angeblich „absoluten" Verfügungsfreiheit von Eigentümer*innen aufzusitzen (die dann wieder auf ein sozialverträgliches Maß zurechtgestutzt werden muss), gehen Immanenztheorien davon aus, dass die Rechte, die Eigentümer*innen legitimerweise zukommen können, von vornherein in ihrem Umfang beschränkt sind. Eigentumsfreiheit muss also immer schon als eine qualifizierte, soziale oder vernünftige, in jedem Fall aber beschränkte Freiheit gedacht werden.

Aber warum und wovon genau wird die Verfügungsfreiheit hier begrenzt? Diese Fragen lassen sich unterschiedlich beantworten. Heuristisch kann man zwischen positivistischen, utilitaristischen und natur- bzw. vernunftrechtlichen Immanenztheorien unterscheiden.

Für das positivistische Eigentumsverständnis sind Inhalt und Schranken des Eigentums gleichursprünglich, weil beide überhaupt erst aus positiver Gesetzgebung resultieren. Sozialpflichtigkeit und Eigentumsschranken treten hier nicht „von außen“ an das Eigentum heran, sondern sind vielmehr eigentumskonstituierend. Das so konstituierte Eigentum ist ein Bündel von Rechten, das die Beziehung zwischen Personen (in Bezug zu einer Sache) regelt (Honoré 1961; Munzer 1990). Dieser Position hat sich u. a. das Bundesverfassungsgericht explizit angeschlossen: „Da es keinen ,absoluten“ Begriff des Eigentums gibt, ist es Sache des Gesetzgebers, Inhalt und Schranken des Eigentums zu bestimmen.“ (BVerfGE 20, 351, 355) ${ }^{10}$ Die rechtliche Position 
von Eigentümer*innen variiert somit von Fall zu Fall; sie ergibt sich aus der „Zusammenschau aller in diesem Zeitpunkt geltenden, die Eigentümerstellung regelnden gesetzlichen Vorschriften“ und damit aus dem gleichrangigen Zusammenspiel von bürgerlichen und öffentlich-rechtlichen Gesetzen (BVerfGE 58, 300, 336; BVerfGE 49, 382, 393). Dadurch fungiert der Gesetzgeber (und insbesondere das öffentliche Recht) nicht mehr primär als Gegenspieler der Freiheit, sondern soll vielmehr gewährleisten, dass das Eigentum seine freiheitliche Funktion überhaupt erst entfalten kann: „Freiheit verkehrt sich keinesfalls in ihr Gegenteil, wenn der Gesetzgeber über ihre Reichweite bestimmt." (Wieland 2013, 30; vgl. auch Habermann 2020)

Für utilitaristische Eigentumstheorien haben Eigentumsrechte instrumentellen Wert und werden von den Zwecken, denen sie dienen, konstitutiv beschränkt. Die wohl einflussreichste Spielart utilitaristischer Eigentumstheorien ist die ökonomische Analyse des Rechts und ihre Theorie der property rights. Diese versteht sich zwar zunächst nicht als normative Theorie, nimmt in der Anwendung aber meist die Form eines ,applied utilitarianism“ an (Posner 1979, 119). Eigentumsrechte sind demnach legitim, weil (bzw. wenn) sie Transaktionskosten und Externalitäten reduzieren und dazu beitragen, Güter denjenigen zugänglich zu machen, für die sie den größten Nutzen haben. War die Theorie in ihren Ursprüngen noch bemüht, auf diesem Wege die absolute Theorie des Eigentums zu rehabilitieren (Coase 1960; Demsetz 1967), so nehmen jüngere Beiträge stärker die marktverzerrenden Wirkungen absoluter Eigentumsrechte in den Blick, was teilweise zu überraschend scharfen Formen von marktradikaler Eigentumskritik geführt hat (vgl. Posner und Weyl 2017). In beiden Fällen aber bleibt der Umfang der Eigentumsrechte zumindest formell durch den behaupteten Zweck der Eigentumsordnung, die Maximierung von Allokationseffizienz, beschränkt.

Für natur- und vernunftrechtliche Eigentumstheorien schließlich basieren die Rechte von Eigentümer*innen auf überpositiven moralischen Ansprüchen, die das Eigentum gleichzeitig begründen und beschränken. ${ }^{11} \mathrm{~Pa}-$ radigmatisch für diesen Ansatz stehen etwa die Eigentumstheorien von John

- spätestens seit dem „Nassauskiesungsbeschluss“ des BVerfG - die herrschende Lehre. Das wird von der totalisierenden Eigentumskritik ignoriert, die stattdessen am überholten Bild eines überpositiven Eigentumsbegriffs der Rechtswissenschaften festhält. Vgl. exemplarisch Nuss (2006, 119, Fn. 8).

11 Damit unterscheiden sie sich von modernen libertären Theorien, die eine absolute Eigentumstheorie vertreten. Vgl. Nozick (1974). 
Locke und Immanuel Kant. Während Ersterer Eigentum auf ein natürliches Recht auf Selbsterhaltung gründet, das sich insbesondere als Recht an den Erträgen der eigenen Arbeit konkretisiert (Locke 2007), versucht Zweiterer das Eigentum unmittelbar aus der praktischen Vernunft abzuleiten (Kant 1998). Beiden Perspektiven ist gemein, dass das so gerechtfertigte Eigentum klaren Grenzen unterliegt: Für Locke ergeben sich aus dem Naturrecht objektiv gültige Aneignungs-, Verfügungs- und Akkumulationsschranken; für Kant bleibt das Eigentumsrecht so lange provisorisch, bis eine globale Rechtsordnung gefunden ist, die vom vereinigten Willen aller gewollt werden kann (vgl. Brandt 1982).

Positivistische, utilitaristische und naturrechtliche Eigentumstheorien verfolgen zwar unterschiedliche argumentative Strategien, können aber in der Sache durchaus übereinstimmen. Beschränkte Eigentumsrechte lassen sich oft auf verschiedenen Wegen begründen, wie sich am Recht, fremde Grundstücke unter gewissen Umständen queren zu dürfen, illustrieren lässt: Positivist*innen führen hier an, dass das Bodeneigentum um seiner selbst willen beschränkt werden muss - wenn ich mein Grundstück nur nutzen kann, wenn ich das Grundstück meines Nachbarn durchquere, dann setzt der Genuss meines Privateigentums voraus, dass jenes meines Nachbarn beschränkt wird (und umgekehrt). Nur den auf einer Insel lebenden Robinson „treffen keine sozialen Pflichten“ (Böhmer 1989, 62). Utilitarist*innen führen die Ineffizienz und kostenintensiven Konflikte ins Feld, die ein generelles Durchquerungsverbot mit sich bringen würde. Und aus dem Naturbzw. Vernunftrecht ließe sich ein Recht auf Bewegungsfreiheit, ein „Jedermannsrecht“" oder Vergleichbares ableiten, etwa mit Kants ,Kugelargument”: Da die Erde eine Kugel ist und die Menschheit somit eine begrenzte Fläche bewohnt, stehen alle Menschen in einer ursprünglichen, vorrechtlichen „Gemeinschaft des Bodens“, aus der einerseits das Eigentum erwächst, andererseits aber auch das „Recht des Erdbürgers [...] die Gemeinschaft mit allen zu versuchen und zu diesem Zweck alle Gegenden der Erde zu besuchen“ (Kant 1998, 175).

Anhand dieser Typologie - einerseits der absoluten Theorie in ihren affirmativen und kritischen Varianten, andererseits der Immanenztheorie in ihren positivistischen, utilitaristischen oder naturrechtlichen Varianten werden wir im Folgenden aktuelle eigentums- und bodenpolitische Konflikte analysieren und kontextualisieren. 


\section{Aktuelle wohnungs- und bodenpolitische Debatten}

Wie eingangs rekonstruiert, besteht die eigentumspolitische Herausforderung der Wohnungskrise für den Gesetzgeber darin, eine sachgerechte Balance zwischen der Privatnützigkeit und der Sozialpflichtigkeit des Eigentums sicherzustellen. Dieser Widerstreit zwischen öffentlichem Interesse und privatem Eigentum ist so alt wie die Städte selbst. Hans Bernoulli schweizerischer Bodenreformer des 20. Jahrhunderts - spitzt diesen Konflikt polemisch zu:

Das Eigentumsrecht widersetzt sich der Erneuerung mit einer Hartnäckigkeit, die etwas Erschreckendes hat: Es ist die Hartnäckigkeit, das unverschämte Rechtsgefühl des in private Hände geratenen Königsvorrechts, es ist die besitzsichere, schadenfrohe Haltung des Monopols. [...] So wie der Liberalismus ohne Gefahr sich entfalten kann, wenn ihm nur erst seine Giftzähne - die Monopole - ausgebrochen sind, so wird auch die Stadtbaukunst gedeihen können, sobald dem Privatmann das ihm so unbedarft zugespielte Monopol - das Grundeigentum - wieder aus den Händen genommen und den Gemeinden zurückgegeben wird. (Bernoulli 1946, 21 und 121)

Damals wie heute liegt das Problem demnach in einer einseitig privatnützig ausgerichteten Eigentums- und Bodenordnung. Aber Bernoullis Zuspitzung unterschlägt, dass auch eine fehlerbehaftete Bodenpolitik der öffentlichen Hand der „Stadtbaukunst“ zuwiderlaufen kann, wie etwa in der massenhaften Ausweisung von Einfamilienhausgebieten in den 1990er und dem Verkauf stadteigener Liegenschaftsportfolios zu Dumpingpreisen in den 200oer Jahren geschehen. ${ }^{12}$ Weder öffentliches Eigentum noch stärkere Eingriffsbefugnisse des Staates gegenüber Eigentümer*innen führen unweigerlich zu lebenswerten Städten. Es gilt also genauer hinzuschauen.

Um das zu tun, gehen wir in diesem Abschnitt dem Streit um die bodenrechtlichen Implikationen der beiden oben eingeführten Dimensionen des Verfügungsrechts - Gebrauch und Verwertung - nach: Wer entscheidet darüber, welchen Zwecken der Boden zugeführt wird? (3.1) Wer soll von der Bodennutzung profitieren? (3.2) Ausgehend von diesen Debatten, bei denen

Auch greift es zu kurz, Planung lediglich als Gegenspielerin zum privaten Eigentum zu verstehen. Das Widerspiel von „Eigentumsfreundlichkeit“ und „Eigentumsfeindlichkeit“ (Davy 1999, 104) räumlicher Planung wird in den nachfolgenden Abschnitten thematisiert. 
das Objekt des Eigentumsrechts im Mittelpunkt steht, wenden wir uns dann der oft vernachlässigten, in der Praxis aber hoch relevanten Frage nach dem Subjekt des Eigentums zu: Welche Akteure können sich überhaupt auf den Eigentumsschutz berufen? (3.3) Wir plädieren dafür, die Schutzwürdigkeit verschiedener Eigentümertypen (z. B. Kleinanleger*innen, gemeinnützigen Genossenschaften, Großkonzernen) differenziert zu betrachten.

\subsection{Eigentum als Recht auf Gebrauch: Der Streit um die Baufreiheit}

Als erste Dimension umfasst Eigentum das Recht, über den Gebrauch einer Sache zu verfügen. Die Frage, wem dieses Gebrauchsrecht zusteht und zu welchen konkreten Handlungen es befugt, äußert sich im Fall städtischen Grundeigentums in der Debatte um die Baufreiheit: Woraus speist sich das Recht, über ein Grundstück zu verfügen und es zu bebauen oder - ebenso wichtig - nicht zu bebauen? Ist die eigentümliche Baufreiheit über den Art. 14 GG garantiert? Wird diese Freiheit verliehen oder beschränkt?

\section{Absolute Theorien: Baufreiheit als originäres Eigentumsrecht}

Für die absolute Eigentumstheorie scheint die Sache klar: Das Gebrauchsrecht liegt zunächst bei den Eigentümer*innen. Schon der frühliberale $\S 65$ des Preußischen Allgemeinen Landrechts von 1794 kannte ein grundsätzlich unbeschränktes, in der Ausübung beschränkbares Eigentumsrecht an Grund und Boden. In dieser „Rechtstradition“ (Papier und Shirvani 2019, Rn. 164f.) hält auch das BVerfG fest: „Das Recht der Bauherrin, ihr Grundstück im Rahmen der Gesetze zu bebauen, ist durch die Eigentumsgarantie des Art. 14 Abs. 1 S. 1 geschützt. "(BVerfGE 35, 263, 276) Diesem Gedanken nach wohnt das Gebrauchsrecht dem Grundeigentum inne und kann allenfalls „von außen“ beschränkt werden. Die Baugenehmigung gilt dann als „Wiedereröffnung“ (Leisner 1992, 1069) eines durch Schrankennormen verschlossenen Raums der grundrechtlichen Freiheit.

Unbestritten ist dabei, dass sich die Dispositionsbefugnisse der Grundeigentümer*innen letztlich auf das „Ob“ und „Wann“ beschränken, kaum aber auf das „Wie“ (Papier und Shirvani 2019, Rn. 165). Die bauliche Nutzbarkeit des Bodens ist in Deutschland flächendeckend über §3o (Bebauungspläne), §34 (unbeplanter Innenbereich) und §35 (Außenbereich) des Baugesetzbuches geregelt. Laut der absoluten Theorie folgt daraus aber nicht, dass es kein originäres Baurecht gebe: Schon der Begriff „Privatnützigkeit“, auf dem das BVerfG sein Verständnis der Eigentumsgarantie aufbaut, und das Wort „Gebrauch“ im Abs. 2 des Art. 14 GG verweisen auf die 
Nutzung des Grundeigentums (Leisner 1992, 1068). Wenn die Bebaubarkeit keinen Teil der Eigentumsgarantie darstellt, dann wäre das Baurecht keine Eigentumsbeschränkung i.S.d. Verfassungsrechts. Der Gesetzgeber würde im grundrechtsfreien Raum manövrieren, er „stünde nicht unter, sondern wäre Herr der Verfassung“ (Depenheuer und Froese 2018, Rn. 43).

Städtebauliche Planung tritt hier dem Eigentum - und der (Bau-)Freiheit - immer schon als Antagonistin entgegen. Diese Argumentation macht sich jedenfalls die Immobilienwirtschaft zu eigen, wenn sie die Verantwortung für die Baulandknappheit bei der fehlenden Kooperationsbereitschaft der Kommunen sucht und eine Eigentumspolitik fordert, die den Wohnbau nicht reguliert, sondern in möglichst unbeschränkter Form ermöglicht (ZIA 2019). Aber auch die totalisierende Eigentumskritik folgt dieser antagonistischen Rhetorik: Weil sowohl die „Freiheit der Beschädigung“ (Nuss) des Eigentums als auch staatlicher Zwang letztlich zu Formen von Unfreiheit führen, sucht die totalisierende Kritik nach herrschaftsfreien Alternativen, etwa in Form von widerständigen Praktiken (Loick 2016) oder nichtproprietären Gegenentwürfen zum Eigentum wie „dem Gemeinsamen“ (Hardt und Negri 2010). Diese Gegenentwürfe bleiben allerdings theoretisch meist unterbestimmt und lassen offen, wie ihre Verrechtlichung konkret aussehen könnte.

\section{Immanenztheorie: Baurecht als Nutzungszuweisung}

Immanenztheorien verfügen über Ressourcen, anders über die Baufreiheit nachzudenken. Besonders deutlich wird das beim Rechtspositivismus, der sich klar gegen die Idee einer abstrakten, vorgesetzlichen Baufreiheit stellt: Der Gesetzgeber schafft mit dem Bebauungsrecht eine Rechtsposition, die nicht durch den Art. 14 Abs. 1 S. 1 GG begründet ist, sondern von außen als eine öffentlich-rechtliche Rechtsposition an das Grundeigentum herantritt (Breuer 1992; Bryde 2012; Wieland 2013). Die Bebaubarkeit ist nur konkret-gesetzlich und apriorisch pflichtgebunden denkbar. Der Eigentumsschutz wirkt dabei nicht direkt, sondern mittelbar: Bestimmt der Gesetzgeber den Inhalt des Eigentums (Art. 14 Abs. 2), bspw. in einem Bebauungsplan, muss er das Institut des Privateigentums ausreichend würdigen. ${ }^{13}$

13 Das Ausklammern von Verfügungsrechten führt demnach auch nicht dazu, dass der Eigentumsschutz ausgehöhlt würde (so Depenheuer und Froese 2018, Rn. 5). Fühlen sich Grundeigentümer*innen benachteiligt, steht der Weg vor die Gerichte offen, um das Gesetz anzufechten. In der Rechtspraxis ist es unerheblich, ob die subjektive Rechtsstellung der Grundeigentümer*in- 
Der Gesetzgeber kann demnach, ohne gegen den Inhalt des Art. 14 GG zu verstoßen, Nutzungsrechte aus der umfassenden Verfügung der Eigentümer*innen ausgliedern. Dies hat das BVerfG im Hinblick auf die Grundwassernutzung und die Bergbauberechtigung bestätigt (BVerfGE 58, 300; $83,201)$. Die grundsätzliche Verfügungsbefugnis als Merkmal des Privateigentums bleibt bewahrt, stellt aber lediglich einen „Richt- und Grenzwert“ (Breuer 1992, Rn. 40) für den Gesetzgeber dar. Eigentümer*innen kommt zwar ein allgemeines Gebrauchsrecht zu, aber nicht notwendig ein Recht „auf diejenige Nutzungsmöglichkeit, die [ihnen] den größtmöglichen wirtschaftlichen Vorteil verspricht“ (BVerfGE 58, 300, 345).

Wird das Baurecht so von außen als öffentlich-rechtliche Eigentumsposition hinzugefügt und damit die Einheitlichkeit des Grundeigentums negiert, dann drängt sich auf, den eingeräumten Eigentumsschutz nach funktionalistischen Kriterien zu differenzieren. Es macht dann bereits für den Umfang originärer Rechte einen Unterschied, ob sich ein Eigentumstitel auf die eigene Krawatte, eine Krawattenfabrik oder den eigenen Vorgarten bezieht. Dieser Unterschied ergibt sich schon aus der Eigenlogik dieser Gegenstände: Weil der Boden - im Gegensatz zu anderen Gütern - zugleich unvermehrbar und unentbehrlich ist, verbietet es sich, „seine Nutzung dem unübersehbaren Spiel der freien Kräfte und dem Belieben des Einzelnen vollständig zu überlassen“" (BVerfGE 21, 73, 82f.).

Wird die Baufreiheit nun verliehen oder beschränkt? Der Immanenztheorie nach wird sie verliehen. In seiner Inhalts- und Schrankenbestimmung zeichnet der Gesetzgeber nicht lediglich „naturalisierte“ Rechte und Pflichten nach, sondern streitet in einer demokratischen Aushandlung über Inhalt und Funktion des Eigentums, ohne sich an einen präpositiven Begriff binden zu müssen. Bodenpolitik und (Bau-)Freiheit stehen daher nicht notwendig in Widerspruch; vielmehr zielt eine effektive städtebauliche Planung auf die Realisierung des Freiheitsversprechens des Eigentums.

\subsection{Eigentum als Recht auf Verwertung: Wem gehört der Profit?}

Nach Berechnungen des ehemaligen Bundesbauministers und Oberbürgermeisters der bayrischen Landeshauptstadt, Hans-Jochen Vogel, stiegen die Baulandpreise in München von 1950 bis 2015 um 34.283 Prozent (Vogel 2018, 23). Diese Bodenrenten werden von der öffentlichen Hand (durch

nen über privates oder öffentliches Recht eingeräumt wurde (Wieland 2013, Rn. 50; Breuer 1992, Rn. 40). 
Planung, soziale und technische Infrastruktur etc.) und der darauf aufbauenden Wirtschaftskraft geschaffen (Löhr 2018a, 2018b). Bodenrenten sind abgabenrechtlich privilegiert und verbleiben - abzüglich der marginalen Grundsteuer und einzelner Abgaben - regelmäßig bei den Grundeigentümer*innen. Ob Boden nun zu Bauland wird, hängt aber nicht von seiner tatsächlichen oder wirtschaftlichen Verfassung, sondern von seiner Rechtslage ab, dem Umstand nämlich, ob der Boden nach §§30, 34 oder 35 BauGB bebaut werden darf (Davy 2000, 66f.). Die planrechtlich bedingte Wertsteigerung schwebt gewissermaßen „wie eine Wolke über der Landschaft und jeder Bodenbesitzer glaubt oder hofft zumindest, diese Wolke werde ihren Segen gerade über seinem Grundstück entladen“ (von Nell-Breuning, zit. in Dransfeld 2018, 136).

Diese privilegierte Position der Grundeigentümer*innen ist nicht das Produkt einer ökonomischen oder gar begrifflichen Notwendigkeit, sondern einer spezifischen Eigentumspolitik. Mit dem Deutschen Juristentag 1972 wurden erstmals Forderungen laut, diese Grundmasche (manche sagen: Webfehler) des Bauplanungsrechts zu berichtigen (Vogel 1972). Forderungen nach dem Planwertausgleich, der seinerzeit im Zentrum der Debatte stand, wurden inzwischen um wertmindernde Positivpflichten, etwa zur Errichtung von Sozialwohnungen, ergänzt (vgl. exemplarisch DIFU und vhw 2017; Münchner Initiative für ein soziales Bodenrecht 2018). Dieser Instrumente, deren ökonomische Effektivität umstritten ist (Löhr 2018a, 35f.), setzen an der Vergemeinschaftung der Bodenrente an. Rechtstheoretisch ausgedrückt handelt es sich dabei um Verkürzungen des Bodeneigentums (Habermann 2020). Der politische Streit um diese Instrumente dreht sich im Kern um die zweite Dimension des Eigentums: Wie weit reicht das Verwertungsrecht beim Bodeneigentum? Wem steht das Recht zu, sich die planbedingten Bodenwertsteigerungen anzueignen?

\section{Wem verbleibt der Bodenwert?}

Wertsteigerungen stehen zunächst denjenigen zu, die sie (auf legitime Weise) geschaffen haben. Dieser allgemeine Leistungsbezug des Eigentums gilt als unkontrovers. Schon in einem seiner ersten Beschlüsse stellt das BVerfG fest, dass es den ,allgemein herrschenden gesellschaftlichen Auffassungen [entspricht], das, was der Einzelne sich durch eigene Leistung [...] erworben hat, im besonderen Sinne als sein Eigentum anzuerkennen und gegenüber Eingriffen als schutzwürdig anzusehen“ (BVerfGE 1, 264, 278). Begründungstheoretisch leitet sich der Leistungsbezug aus der freiheitssichernden 
Funktion des Eigentums ab: Für meine frei gewählten Handlungen trage ich nicht nur die Verantwortung, sondern bin ebenso berechtigt, von ihren Erträgen zu profitieren. ${ }^{14}$

Aber je nach Eigentumsverständnis lassen sich aus dem Kriterium des Leistungsbezugs unterschiedliche Positionen im Bodenrecht ableiten. Folgt man der absoluten Eigentumstheorie, die die Baufreiheit als Teil der Eigentumsgarantie versteht, dann gelten alle planerischen Verbote und Pflichten als Freiheitsbeschränkung (s.o.). Werden diese Beschränkungen beseitigt und die Baufreiheit wiederhergestellt, steht der eintretende Mehrwert allein den Eigentümer*innen zu (Leisner 1992, 1066). Die Einheitlichkeit des Eigentums begründet demnach einen pauschalen Anspruch auf den generierten Mehrwert und verbietet einen differenzierten Leistungsbezug (Burgi 2020, 264). Da die planungsrechtliche Eröffnung der Bebaubarkeit keine staatliche Nutzungsgewährung darstellt, ist die Baugenehmigung auch keine Wertzugabe durch die öffentliche Hand, nach der sich eine Wertabschöpfung aufdrängt (Papier und Shirvani 2019, Rn. 592). Der Staat kann allenfalls Kosten eigener, gezielt erbrachter Leistungen, wie etwa die infrastrukturelle Erschließung, auf die Begünstigten umlegen, aber das Recht auf konjunkturell oder planbedingte Wertsteigerungen verbleibt bei den Eigentümer*innen.

Für diese Position werden zwei weitere Argumente geltend gemacht:

(1) Eine Leugnung der Baufreiheit würde zu einer massiven Entwertung des Bodens führen. Entstünde das Verwertungsrecht für die bauliche Nutzung allein durch eine konstitutive Zuweisung, dann wäre bei Eigentumseingriff auch nur das zu entschädigen, worauf sich die eigentümliche Nutzungsfreiheit erstreckt. Es verbliebe allenfalls ein agrarischer Bodenwert. Das aber würde dem Art. 14. Abs. 3 GG widersprechen, der die Enteignung nach dem Verkehrswert verlangt, welcher sich ganz wesentlich auf die Baulandqualität und Renditeerwartung erstreckt (Leisner 1992, 1072).

(2) Planwertgewinne stellen nicht zwingend das Resultat staatlicher Investitionen dar. Sie können ebenso auf Eigenleistungen basieren oder sich als inflationsbedingte Scheingewinne erweisen (Papier und Shirvani 2019,

14 Es gibt verschiedene eigentumstheoretische Möglichkeiten, dieses Begründungsverhältnis konkret auszubuchstabieren - etwa als vorvertragliches Verwertungsrecht an der eigenen Arbeit (Nozick 1974; Locke 2007), als für personale Identität notwendige „äußere Sphäre der Freiheit“ (Hegel 1986), als Ergebnis eines unhintergehbaren kulturell-zivilisatorischen Lernprozesses (Hayek 2013) oder als ökonomische Anreizfunktion (Demsetz 1967). 
Rn. 594). Und selbst wo sie auf den Staat zurückzuführen sind, sind sie oft keine Wertzugabe durch die öffentliche Hand, sondern vielmehr Zeichen einer falschen, weil zu restriktiven Eigentumspolitik. So sind es oft nicht die Planungsprozesse selbst, „sondern eine der Eigentumsinstitutsgarantie des Art. 14 Abs. 1 S. 1 nicht hinreichend Rechnung tragende planerische Inaktivität oder Säumigkeit der Kommunen, die die gravierenden Wertzuwächse bedingen“ (ebd., Rn. 592, vgl. auch Depenheuer und Froese 2018, Rn. 301).

Folgt man hingegen einer Immanenztheorie des Eigentums, so lässt sich dagegenhalten: Ebenso wie Eigentümer*innen kein Recht auf die maximale Verwertung des Bodens zusteht (s.o.), besteht auch kein Anspruch auf die Inwertsetzung bzw. Werterhöhung durch planerische und infrastrukturelle Maßnahmen (Bryde 2012, Rn. 65). Weil eine unregulierte Bebauung gerade nicht zu einer nachhaltigen Stadt- und Wertentwicklung beiträgt, sondern dieser zuwiderlaufen würde, stellt das Bau- und Planungsrecht nicht per se einen restriktiven Eingriff in das Verwertungsrecht der Eigentümer*innen dar. Es zielt vielmehr darauf, dieses sinnvoll zu ermöglichen. Ebenso wie die Gebrauchsrechte sind auch die Verwertungsrechte des Eigentums daher nur apriorisch pflichtgebunden denkbar. Das Bau- und Planungsrecht wird so zur Wertzugabe durch die Gemeinschaft.

Damit begegnet die Immanenztheorie den beiden o.g. Argumenten der absoluten Theorie wie folgt:

Ad (1): Wenn ein überhöhter Bodenpreis zuvorderst auf den verliehenen Gebrauchsrechten beruht, dann sind auch deutlich reduzierte Enteignungsentschädigungen oder Vorkaufsrechte unter dem Verkehrswert denkbar. Die Eigentumsgarantie „gebietet [...] nicht, einmal ausgestaltete Rechtspositionen für alle Zukunft in ihrem Inhalt unangetastet zu lassen“ (BVerfGE 83, 201, 212), sondern, dass die Privatnützigkeit gewahrt bleibt, d.h., dass das Objekt angemessen nutzbar und verwertbar ist. ${ }^{15}$ Bis zu dieser Grenze ist das Einbehalten von Bodenwerten legitim und daran zu messen, auf wessen Leistung die Wertsteigerung zurückgeht. Je mehr sie „staatsverursacht bzw. der Allgemeinheit zuzurechnen ist, desto eher können bzw. müssen Abzüge gemacht werden“ (Bryde 2012, Rn. 90; siehe auch Weigelt 2016, 77).

15 Die dogmatische Grundlage liegt im Grundsatz der Verhältnismäßigkeit: Die Inhalts- und Schrankenbestimmung darf „nicht zu einer übermäßigen Belastung führen und den Eigentümer im vermögensrechtlichen Bereich unzumutbar treffen“" (BVerfGE 58, 137, 148). 
Ad (2): Die Annahme Papier und Shirvanis (2019), dass durch eine Ausweitung der Baulandmenge der Bodenpreis fällt, stellt einen Trugschluss dar. Ob Boden Baulandqualität erlangt, hängt von seiner rechtlichen Qualität ab (Davy 1999; 2000). Der Preis des Bodens bemisst sich also zuvorderst am planungsrechtlichen Status, d.h. an der verbindlichen Zuweisung von Gebrauchsrechten. Wenn der Bodenwert von dem rechtlichen Status des Bodens abhängt, dann müssen eigentumsgestaltende Instrumente auch dort ansetzen, um den Bodenpreis zu dämpfen. ${ }^{16}$

Wem verbleibt also der Profit? Der aus der absoluten Eigentumstheorie generierte, pauschale Anspruch auf Bodenwertsteigerungen erweist sich als argumentativ nicht haltbar. Nicht die Kommune muss sich mit dem Einhalten solcher Bodenwerte, die ihr durch gezielt erbrachte Maßnahmen zustehen, begnügen, sondern umgekehrt: das Verwertungsrecht der Grundeigentümer*innen ist daran zu bemessen, inwiefern sie die Erträge kraft eigener Leistung erlangt haben.

\subsection{Wer genießt den Eigentumsschutz?}

Die Immanenztheorie stellt dem Gedanken einer zunächst absoluten, im Nachgang durch den Gesetzgeber eingeschränkten Freiheit eine von vornherein qualifizierte, pflichtige Freiheit entgegen. Sowohl bei der Baufreiheit als auch bei der Frage nach den planbedingten Wertsteigerungen werden die dem Eigentum innewohnenden Rechte und Pflichten von der Objekt-Seite der Eigentumsrelation hergeleitet. Im nun folgenden Abschnitt erweitern wir dieses Bild um einen weiteren, oft vernachlässigten Aspekt: Welche Arten von Subjekten sollte das subjektive Recht des Eigentums schützen? Konkret: Sollte es für die Frage nach der Schutzwürdigkeit des Eigentums einen grundsätzlichen Unterschied machen, ob eine Immobilie von einer profitori-

16 Nach Davy (1999; 2000) fallen die Rollen des Produzenten und des Anbieters am deutschen Bodenmarkt auseinander: Die Kommune produziert durch ihre hoheitliche Planung Bauland, das die Grundeigentümer*innen auf dem Bodenmarkt anbieten können. In der Folge treten Baulandüberschuss und ein Mangel an verfügbaren Baugrundstücken mitunter simultan auf („Baulandparadoxon“). „Eine Absenkung des allgemeinen Bodenpreisniveaus wäre höchstens zu erwarten, wenn ein Vielfaches der benötigten Baulandmenge ausgewiesen und erschlossen wird - eine unvertretbare Vergeudung von Boden, die gegen die Bodenschutzklausel des §1a Abs. 1 BauGB verstieße“ (2000, 68). Siehe zu Flächenverfügbarkeit und Bodenpreisen auch Hoffmann-Axthelm (1993). 
entierten Aktiengesellschaft, einer Erbengemeinschaft, privaten Vermietern oder einer Wohneigentümerin besessen wird?

Eine Antwort muss sich am Art. 19 Abs. 3 GG messen lassen: „Die Grundrechte gelten auch für inländische juristische Personen, soweit sie ihrem Wesen nach auf diese anwendbar sind." Die entscheidende Frage lautet also: Sind Eigentumsrechte an Wohnungen und Immobilien ihrem Wesen nach auf alle natürlichen und inländischen juristischen Personen gleichermaßen anwendbar? Die Grundgesetzkommentator*innen beantworten diese Frage - in der Sache weitgehend unbegründet und in der Breite überraschend - mit Ja (Depenheuer und Froese 2018, Rn. 189; Bryde 2012, Rn. 8; Papier und Shirvani 2019, Rn. 331; Wieland 2013, Rn. 84).

Überraschend ist diese Pauschalität vor allem aufgrund der engen Verknüpfung von Eigentum und Freiheit, die den Dreh- und Angelpunkt unserer Eigentumsordnung darstellt: „Der Eigentumsgarantie kommt im Gefüge der Grundrechte insbesondere die Aufgabe zu, dem Träger des Grundrechts einen Freiheitsraum im vermögensrechtlichen Bereich zu sichern.“ (BVerfGE 31, 229, 240) Je mehr ein Eigentumstitel dem Schutz von Existenz, Freiheit und Persönlichkeitsentwicklung der Eigentümer*in zugutekommt, desto stärker muss er vor Verlust und Substanzgefährdung geschützt werden. ${ }^{17}$

Zumindest bei natürlichen Personen leitet die Rechtsprechung daraus eine differenzierte Haltung ab: Wenn ein Grundstück „zu dauerhaftem Wohnen genutzt wird, und damit gewachsene soziale Beziehungen der Eigentümer zu ihrem auch örtlich geprägten Umfeld“ ermöglicht, sind Beschränkungen des Grundrechts besonders streng zu prüfen (BVerfGE 134, 242, 290; vgl. auch Davy 2006, 60) - nicht zuletzt, weil bei Kleineigentümer*innen die kumulierte Wirkung von Handlungsverpflichtungen schnell dazu führen kann, dass die Belastungen in der Summe unzumutbar sind (Burgi 2020; vgl. auch Weigelt 2016, 361f.).

Inwiefern lässt sich nun sagen, dass Eigentumstitel auch für juristische Personen eine freiheits- und existenzsichernde Funktion haben? Gemäß der sogenannten Durchgriffstheorie können juristischen Personen nur dann Grundrechte nach Art. 14 GG zugesprochen werden, wenn diese als

17 Die Kritik, dass der Genuss von Grundeigentumsrechten stark ungleich verteilt ist, schlägt hier fehl, da die „Freiheitsformel“ auch zur Ausweitung des Grundrechtsschutzes auf vermögenswerte Positionen dient, etwa zur Anerkennung des „Besitzrechtes des Mieters“ als eine durch die Art. 14 Abs. 1 S. 1 geschützte Eigentümerstellung (BVerfGE 89, 1). 
Ausdruck der freien Entfaltung einer oder mehrerer natürlicher Personen gelten können (BVerfGE 143, 246, 313f. m.w.N.), die freiheits- und existenzsichernden Funktionen des Eigentums also auf natürliche Personen durchgreifen. Wenn die Analogie zur natürlichen Person halten soll, wäre auch hier eigentlich eine differenzierte Position zu erwarten; die Rechte persönlich involvierter Kleineigentümer*innen müssten als besonders schützenswert, hochkonzentriertes oder spekulativ genutztes Eigentum hingegen als weniger schützenswert gelten. Polemisch zugespitzt: Der Freiheitsgewinn, den mir das zweiundsiebzigste Haus in meinem über ein Steuerparadies verwalteten Immobilienportfolio sichert, dürfte in der Regel geringer ausfallen als der, den dasselbe Haus als Eigenheim für seine Bewohner*innen bedeuten würde. ${ }^{18}$ Auch das BVerfG scheint diese Einsicht inzwischen grundsätzlich zu teilen, wenn es auf den Unterschied zwischen privaten und anderen gewerblichen Vermieter*innen, etwa Wohnungsunternehmen, hinweist, „deren Eigentum nur in geringem Maße der persönlichen Freiheit eines Einzelnen dient“ (BVerfG, 3. Kammer des Ersten Senats, Beschl. v. 18.7.2019, Rn. 70).

Ein eindrückliches Bespiel für die Verwerfungen einer Eigentumsordnung, die juristische und natürliche Personen pauschal gleichsetzt, liefert das Phänomen des beneficial ownership. Hier halten Individuen Eigentumstitel nicht direkt, sondern verwalten sie über eine undurchsichtige Reihe von Briefkastenfirmen. ${ }^{19}$ Beneficial owners üben ihre Eigentumsrechte somit anonym aus, d.h., sie schöpfen Profite ab oder verfügen über den Gebrauch des Eigentums, ohne für mögliches Fehlverhalten oder Folgekosten zur Rechenschaft gezogen werden zu können. Das aber hebelt die Institutionen einer freiheitlichen Eigentumsordnung systematisch aus: Erstens schafft beneficial ownership die Möglichkeit, Grundstücke am regulären Immobilienmarkt vorbei zu handeln, indem nicht die Grundstücke selbst übertragen werden, sondern Anteile an den juristischen Personen, die als Grundstückseigentümer*innen eingetragen sind (share deals, vgl. Thurn 2020). Damit werden bestehende eigentumspolitische Instrumente wie Grundbuchände-

18 So auch Davy (2006, 60): „[E]inen besonderen Freiheitsgewinn ziehen juristische Personen aus ihrem Immobilienvermögen wohl selten."

19 Wie Guy Shrubsole (2019) für das Vereinigte Königreich zeigt, handelt es sich bei den anonymen Eigentümer*innen keinesfalls um ein Nischenphänomen: Seinen Recherchen zufolge sind etwa 17 Prozent des Bodens in anonymem Privatbesitz. Den Hinweis auf das Phänomen beneficial ownership verdanken wir Eva Weiler. 
rungen, Grunderwerbssteuern oder gemeindliche Vorkaufsrechte faktisch außer Kraft gesetzt. Die entstehende Intransparenz öffnet, zweitens, kriminellen Praktiken wie Geldwäsche und Steuerhinterziehung Tür und Tor (OECD 2019). Und drittens produziert beneficial ownership soziale Folgekosten, weil es Dritten erschwert oder unmöglich gemacht wird, bestehende Eigentumsstrukturen zu durchschauen und auf diese Strukturen adäquat zu reagieren. Das erodiert nicht nur ihre Rechtssicherheit und politische Handlungsmacht, sondern ist auch ökonomisch ineffizient (vgl. dazu Merrill und Smith 2000, 24f.).

Beneficial owners sind keine Parasiten, die eine ansonsten funktionale Eigentumsordnung missbrauchen, sondern vielmehr Ausdruck ihrer dysfunktionalen Normalität. Denn um juristische Personen eigentumsrechtlich mit natürlichen Personen gleichstellen zu können, muss man die Differenz zwischen beiden ignorieren. Diese Differenz besteht darin, dass natürliche Personen in einem unmittelbaren, begrifflich notwendigen Sinne frei sind, während juristische Personen nur in einem vermittelten Sinne als frei gelten können; oder in kantischer Formulierung: Natürliche Personen sind Selbstzwecke, juristische Personen hingegen Mittel zum Zweck. Im Gegensatz zu den Grundrechten natürlicher Personen sind die ,Rechte juristischer Personen daher allenfalls mittelbar freiheitsverbürgend: Sie greifen nicht automatisch auf natürliche Personen durch. Dann aber ist es problematisch, juristische und natürliche Personen eigentumsrechtlich pauschal gleichzustellen.

Aus ebendiesem Grund hatte noch Kant das Eigentumsrecht für juristische Personen verneint und bspw. die Enteignung von Klöstern und Ritterorden explizit verteidigt. Diesen stünden bei Enteignung zwar gewisse Entschädigungen zu, aber sie könnten „nicht klagen, daß ihnen ihr Eigenthum genommen werde; denn der Grund ihres bisherigen Besitzes lag nur in der Volksmeinung und mußte auch, solange diese fortwährte, gelten. Sobald diese aber erlosch, [...], so mußte [...] das vermeinte Eigenthum aufhören.“ (Kant 1998, 147; ähnlich auch Hegel 1986, §46 Zusatz). Damit weist Kant den Eigentumsanspruch juristischer Personen nicht pauschal zurück, schließlich gibt es Situationen, in denen juristische Personen nützliche Instrumente darstellen. Er weist ihm aber einen fundamental anderen Status zu als dem Eigentum natürlicher Personen. Während letzteres seine Grundlage in der menschlichen Vernunft hat und insofern unbedingt schützenswert ist (man könnte auch sagen: ein Grundrecht darstellt), wird das Eigentum juristischer Personen nur durch menschliche Konvention geschaffen und kann durch diese auch ebenso gut wieder genommen werden. In den Worten von 
Franz Neumanns Aktualisierung des Arguments: „Wenn das Eigentum der Freiheit dienen soll und wenn die Freiheit nur dem Menschen zukommt, so kann korporatives Eigentum, ob nun sozial notwendig oder nicht, niemals für sich in Anspruch nehmen, ein Grundrecht gleichen Ranges [...] zu sein.“ (Neumann 1967, 123)

Wer sollte also den Eigentumsschutz genießen? Nur solche Entitäten, für die das Eigentum seine freiheits- und existenzsichernde Funktion erfüllen kann. Der Gesetzgeber steht hier in der Pflicht, der Durchgriffstheorie Geltung zu verschaffen, was weit mehr erfordert, als nur die Lücke zwischen natürlichen und juristischen Eigentümer*innen durch Post-hoc-Maßnahmen nominell zu verkleinern. ${ }^{20}$ Die Unterscheidung zwischen Eigentumsformen verlangt auch nach verschiedenen Berechtigungen und Verpflichtungen, etwa in Form von differenzierten Entschädigungs- und Kaufpreisbemessungen (bei Enteignungen und Vorkaufsrechten) sowie hinsichtlich der Zuweisung von Bauland oder der steuerlichen Belastung. Das Wohnungsgemeinnützigkeitsgesetz, das 1990 abgeschafft wurde, gegenwärtig aber wieder Zuspruch erhält (Deutscher Bundestag 2020), bietet vielversprechende Differenzierungskriterien. Es verstößt nicht gegen die freiheitliche Grundordnung, sondern dient gerade ihrer Aufrechterhaltung, gemeinnützige Eigentumsformen gegenüber solchen zu privilegieren, die primär ökonomische Zwecke verfolgen.

\section{Schluss: Eigentumspolitische Spielräume nutzen!}

Auf die „soziale Frage unserer Zeit“ (Seehofer), die Wohnungsfrage, gibt es keine einfachen Antworten. Zunächst einmal gilt es, die Frage überhaupt richtig zu stellen. Unserer Diagnose nach kreisen gegenwärtige Debatten um bezahlbaren Wohnraum, Wohnungsnot, Mietpreisbremsen oder „gierige“ Immobilienkonzerne vorrangig um Oberflächenphänomene, ohne die eigentlichen Ursachen der Wohnungsnot hinreichend in den Blick zu bekommen. Die Wohnungskrise ist eigentlich eine Bodenkrise und diese ist das Produkt einer spezifischen Eigentums- und Bodenpolitik.

Ausgehend von der Gegenüberstellung von absoluter und immanenter Eigentumstheorie haben wir drei eigentumspolitische Konfliktlinien

20 Wie etwa mit dem 2017 eingeführten ,Transparenzregister. Trotz dieser Maßnahme bleibt bspw. auf dem Berliner Wohnungsmarkt ein Drittel der finalen wirtschaftlichen Berechtigten hinter Objektgesellschaften unbekannt. Siehe dazu Trautvetter und Henn (2020). 
nachgezeichnet, denen die titelgebende Frage „Wem gehört die Stadt?“ ihr Gewicht verdankt - den Streit um die Baufreiheit (3.1), um Bodenwertsteigerungen (3.2) und um die Reichweite des Eigentumsschutzes (3.3). Während die absolute Theorie Gebrauchs- und Verwertungsrecht als originäre, konstitutive Rechte von Eigentümer*innen betrachtet, die allenfalls von außen eingeschränkt werden können, wartet die Immanenztheorie mit einem apriorisch pflichtgebundenen Eigentumsverständnis auf.

Diese Verschränkung von Freiheit und Pflicht ermöglicht den Übergang von der wenig überzeugenden Gegenüberstellung „freie private Verfügung vs. freiheitsbeschränkende öffentliche Planung“ zu einem Gebrauchsrecht als Nutzungszuweisung (3.1) und einem differenzierten Leistungsbezug bei der Verwertung von Böden (3.2). Die Primärfunktion des Eigentums natürlichen Personen einen „Freiheitsraum“ zu gewähren - spricht dabei nicht für eine pauschale Ausdehnung des Eigentumsschutzes auf alle juristischen Personen, sondern für eine differenzierte Schutzwürdigkeit verschiedener Akteure (3.3).

Wem gehört nun die Stadt, wie also sähe eine angemessene eigentumspolitische Rahmung des urbanen Boden- und Wohnungsmarktes aus? Weder die absolute Eigentumstheorie noch die totalisierende Eigentumskritik haben aus unserer Sicht überzeugende Antworten anzubieten. Denn weder ein in Stein gemeißeltes, allenfalls nachträglich beschränkbares Privateigentum noch die Suche nach radikal neuen Institutionen scheinen geeignet, den komplexen Anforderungen einer Wohnungs- bzw. Bodenkrise im Rahmen einer freiheitlichen Eigentumsordnung beizukommen. Anstatt Eigentum zu verabsolutieren oder zu verwerfen, gilt es daher, das Eigentumsrecht, wie es auch das BVerfG fordert, fortwährend an die gesellschaftlichen und wirtschaftlichen Verhältnisse anzupassen (BVerfGE 31, 229, 240). Angesichts einer grassierenden städtischen Wohnungs- und Bodenkrise, in der die Freiheit Einzelner nicht mehr (nur) vor staatlichen Übergriffen, sondern vor allem auch vor den Folgen ökonomischer Ungleichheiten geschützt werden muss, stehen Gesetzgeber und Kommunen in der Pflicht, sich ihrer eigentumspolitischen Spielräume zu bedienen. Eine progressive Eigentumspolitik täte gut daran, mit dem Leistungsbezug und der freiheits- und existenzsichernden Funktion des Eigentums dessen immanente Normen konsequent im Blick zu behalten. 


\section{Literatur}

Anders, Sascha, Stefan Kreutz und Thomas Krüger. 2020. „Corona und die Folgen für die Innenstädte“. Informationen zur Raumentwicklung Nr. 4 (2020): 56-67.

Arbeitskreis der Oberen Gutachterausschüsse, Zentralen Geschäftsstellen und Gutachterausschüsse in der Bundesrepublik Deutschland (AKOGA). 2019. Immobilienmarktbericht Deutschland 2019. Oldenburg.

Bauman, Zygmunt. 2018. Stadt der Ängste, Stadt der Hoffnungen. Wien: Edition Konturen.

Bernoulli, Hans. 1946. Die Stadt und ihr Boden. Zürich: Verlag für Architektur.

Blackstone, William. 2016 [1766]. The Oxford Edition of Blackstone's: Commentaries on the Laws of England. Oxford University Press.

Böhmer, Werner. 1989. Eigentum aus verfassungsrechtlicher Sicht. In Das Eigentum, hg. von Karl-Dietrich Erdtmann und Jürgen Baur, 31-89. Göttingen: Vandenhoeck \& Ruprecht.

Brandt, Reinhard. 1982. „Das Erlaubnisgesetz, oder: Vernunft und Geschichte in Kants Rechtslehre“. In Rechtsphilosophie der Aufklärung. Symposium Wolfenbüttel, hg. v. dems., 223-285. Berlin: de Gruyter.

Breuer, Rüdiger. 1992. „£ 42 BauGB“. In Baugesetzbuch Kommentar, hg. von Rüdiger Breuer und Hans Schrödter, 870-917. 5. Auflage. München: Verlag Franz Vahlen.

Bryde, Brun-Otto. 2012. „Artikel 14“. In Grundgesetz Kommentar, hg. von Philip Kunig, Andreas von Arnauld und Ingo von Münch, 1133-1202. 6. Auflage. Bd. 1. München: C.H. Beck.

Bundesinstitut für Bau-, Stadt- und Raumforschung (BBSR). 2021. Wohnungs- und Immobilienmärkte. Stand: November 2020. Bonn.

Bunzel, Arno, und Eva Maria Niemeyer. 2018. „Der Gesetzgeber ist beim Bodenrecht gefordert“. Zeitschrift für deutsches und internationales Bau- und Vergaberecht, Nr. 8: 743-751.

Burgi, Martin. 2020. „Eigentumsordnung und Wohnungsnot: Spielräume für eine wohnraumbezogene Bodenpolitik“. NVwZ: 257-264.

CDU, CSU und SPD. 2018. Ein neuer Aufbruch für Europa. Eine neue Dynamik für Deutschland. Ein neuer Zusammenhalt für unser Land. Koalitionsvertrag zwischen CDU, CSU und SPD. Koalitionsvertrag der 19. Legislaturperiode. Berlin.

Coase, R.H. 1960. „The Problem of Social Cost“. Journal of Law \& Economics, 1-44.

Davy, Benjamin. 1999. „Boden und Planung - Zwischen Privateigentum und Staatsintervention“. In Was ist Raumplanung? Dortmunder Beiträge zur Raumplanung (Band 89), 101-122. Dortmund: Institut für Raumplanung der Universität Dortmund. 
Davy, Benjamin. 2000. „Das Bauland-Paradoxon: Wie planbar sind Bodenmärkte?“. In Regionale Koordination der Baulandausweisung, hg. v. Klaus Einig, 61-78. Berlin: Verlag für Wissenschaft und Forschung.

Davy, Benjamin. 2006. Innovationspotentiale für Flächenentwicklung in schrumpfenden Städten am Beispiel Magdeburg. Dortmund: Lehrstuhl für Bodenpolitik, Bodenmanagement, kommunales Vermessungswesen der Fakultät Raumplanung/Universität Dortmund.

Davy, Benjamin. 2018. „After form. The credibility thesis meets property theory“. Land Use Policy Nr. 79: 854-862.

Demsetz, Harold. 1967. „Toward a Theory of Property Rights“. The American Economic Review, Vol. 57, No. 2, 347-359.

Depenheuer, Otto, und Judith Froese. 2018. „Artikel 14“. In Grundgesetz Kommentar, hg. von Hermann von Mangoldt, Peter M. Huber, Friedrich Klein, Christian Starck, Andreas Voßkuhle, 1359-1541. 7. Auflage. Bd. 1. München: C.H. Beck.

Deutscher Bundestag. 2019a. Stenografischer Bericht der 137. Sitzung, 19. Wahlperiode. Plenarprotokoll 19/137. Berlin.

Deutscher Bundestag. 2019b. Wohngeld- und Mietenbericht 2018. Drucksache 19/11750. Berlin.

Deutscher Bundestag. 2020. Entwurf eines Gesetzes zur neuen Wohngemeinnützigkeit, Drucksache 9/17307. Berlin.

Deutsches Institut für Urbanistik und vhw - Bundesverband für Wohnen und Stadtentwicklung e.V. (DIFU und vhw). 2017. Bodenpolitische Agenda 2020-2030. Warum wir für eine nachhaltige und sozial gerechte Stadtentwicklungs- und Wohnungspolitik eine andere Bodenpolitik brauchen. Berlin.

Dransfeld, Egbert. 2018. „Kommunale Boden- und Liegenschaftspolitik. Wohnbaulandstrategien und Baulandbeschlüsse auf dem Prüfstand“. vhw Forum Wohnen und Stadtentwicklung Nr. 3: 136-140.

Garnsey, Peter. 2007. Thinking about Property, Cambridge: Cambridge University Press.

Habermann, Tobias. 2020. „Dirigistischer Eingriff oder Eigentumsverkürzung. Bodenpolitik zwischen vorpositivem und positivistischem Eigentum“. Raumplanung Nr. 2/3: 206-211.

Hardt, Michael, und Antonio Negri. 2010. Common Wealth: Das Ende des Eigentums, Frankfurt: Campus.

Harvey, David. 2008. „The Right to The City“, New Left Review 53, 28-40.

Haus \& Grund: „Haus \& Grund: Ausweitung der Vorkaufsrechte gängelt Eigentümer“. Pressemittelung vom 2.7.2019, https://www.hausundgrund.de/haus-grund-ausweitung-der-vorkaufsrechte-gaengelt-eigentuemer, zugegriffen zuletzt am 19.7. 2020.

Hayek, Friedrich August. 2013. Law, legislation and liberty. A new statement of the liberal principles of justice and political economy. London: Routledge. 
Hegel, Georg Wilhelm Friedrich. 1986. Grundlinien der Philosophie des Rechts, Frankfurt am Main: Suhrkamp.

Helbig, Marcel, und Stefanie Jähnen. 2018. Wie brüchig ist die soziale Architektur unserer Städte? Trends und Analysen der Segregation in 74 deutschen Städten. Discussion Paper des Wissenschaftszentrum Berlin für Sozialforschung. Berlin.

Held, Tobias, und Matthias Waltersbacher. 2015 "Wohnungsmarktprognose 2030“. BBSR-Analysen KOMPAKT, Nr. 7: 1-20.

Hoffmann-Axthelm, Dieter. 1993. Die dritte Stadt. Frankfurt am Main: Suhrkamp.

Holm, Andrej, Henrik Lebuhn, Stephan Junker und Kevin Neitzel. 2018. Wie viele und welche Wohnungen fehlen in deutschen Großstädten? Die soziale Versorgungslücke nach Einkommen und Wohnungsgröße. Working Paper Forschungsförderung Nr. 63. Düsseldorf.

Holm, Andrej. 2011. „Wohnung als Ware: zur Ökonomie und Politik der Wohnungsversorgung“. Widersprüche: Zeitschrift für sozialistische Politik im Bildungs-, Gesundheits- und Sozialbereich 31(121), 9-20.

Honoré, Anthony Maurice. 1961. „Ownership“. In Oxford essays on jurisprudence, herausgegeben von Anthony Gordon Guest, 106-147. Oxford: Oxford University Press.

Hubeli, Ernst 2019. Die neue Krise der Städte. Zur Wohnungsfrage im 21. Jahrhundert. Zürich: Rotpunktverlag.

Kant, Immanuel. 1998. Metaphysische Anfangsgründe der Rechtslehre, Hamburg: Meiner.

Knoll, Katharina, Moritz Schularick und Thomas Steger. 2017. No Price Like Home. Global House Prices, 1870-2012. Cesifo Working Paper No. 5006. München: Center for Economic Studies und Info Institute.

Lebuhn, Henrik, und Andrej Holm. 2017. Wohnverhältnisse in Deutschland - eine Analyse der sozialen Lage in 77 Großstädten. Bericht aus dem Forschungsprojekt „Sozialer Wohnversorgungsbedarf" der Hans Böckler Stiftung. Berlin, Düsseldorf.

Lefebvre, Henri. 2016 [1968]. Das Recht auf Stadt. Hamburg: Edition Nautilus.

Leisner, Walter. 1992. „Baufreiheit oder staatliche Baurechtsverleihung?“, Deutsches Verwaltungsblatt Nr. 16: 1065-1072.

Locke, John. 2007. Zweite Abhandlung über die Regierung. Frankfurt am Main: Suhrkamp.

Löhr, Dirk. 2018a. „Wege und Irrwege der aktuellen Bodenreformdiskussion“. Zeitschrift für Sozialökonomie Nr. 196/197: 31-45.

Löhr, Dirk. 2018b. „Boden - die verkannte Umverteilungsmaschine“. Zeitschrift für Sozialökonomie Nr. 198/199: 3-19.

Loick, Daniel. 2016. Der Missbrauch des Eigentums. Berlin: August.

Merrill, Thomas, und Henry Smith. 2000. „Optimal Standardization in the Law of Property: The Numerus Clausus Principle“. The Yale Law Journal 110(1), 1-70. 
Mill, John Stuart. 2016 [1871]. Grundsätze der politischen Ökonomie. Marburg: Metropolis.

Münchner Initiative für ein soziales Bodenrecht. 2018. Kommunaler Impuls zu einer gemeinwohlorientierten Bodenpolitik. München.

Munzer, Stephan. 1990. A theory of property. Cambridge: Cambridge University Press.

Neumann, Franz. 1967. Demokratischer und autoritärer Staat. Studien zur politischen Theorie. Frankfurt am Main: Europäische Verlagsanstalt.

Nozick, Robert. 1974. Anarchy, State, and Utopia. Oxford: Blackwell.

Nuss, Sabine. 2006. Copyright \& Copyriot. Aneignungskonflikte um geistiges Eigentum im informationellen Kapitalismus. Münster: Westfälisches Dampfboot.

Nuss, Sabine. 2019. Keine Enteignung ist auch keine Lösung. Die große Wiederaneignung und das vergiftete Versprechen des Privateigentums. Berlin: Dietz.

OECD. 2019. A Beneficial Ownership Implementation Toolkit, https://www.oecd. org/tax/transparency/beneficial-ownership-toolkit.pdf, zugegriffen zuletzt am 19.7.2020.

Papier, Jürgen, und Foroud Shirvani. 2019. „Art. 14“. In Grundgesetz Kommentar, herausgegeben von Theodor Maunz und Günter Dürig. Loseblatt-Kommentar. 90. Ergänzungslieferung. München: Beck-Online.

Penner, James E. 1996. „The „Bundle of Rights' Picture of Property“. UCLA Law Review 43(3), 711-820.

Posner, Eric, und Glen Weyl. 2017. „Property Is Only Another Name For Monopoly“. Journal of Legal Analysis 9, 51.

Posner, Richard. 1979. „Utilitarianism, Economics, and Legal Theory“. The Journal of Legal Studies 8(1), 103-140.

Prantl, Heribert. 2018. „Mit dem Boden fängt es an“. Süddeutsche Zeitung, 21.9.2018.

Proudhon, Pierre-Joseph. 2018. Was ist das Eigentum? Münster: Unrast.

Robert Koch Institut (RKI). 2015. Infektionsschutz und Infektionsepidemiologie. Fachwörter - Definitionen - Interpretationen. Berlin.

Rötzer, Florian. 2020. Sein und Wohnen: Philosophische Streifzüge zur Geschichte und Bedeutung des Wohnen. Frankfurt: Westend.

Schermaier, Martin. 2017. „Dominus actuum suorum. Die willenstheoretische Begründung des Eigentums und das römische Recht“. Zeitschrift der Savigny-Stiftung für Rechtsgeschichte: Romanistische Abteilung, Band 134, Heft 1, 49-105.

Senatsverwaltung für Stadtentwicklung und Wohnen (SenStadt Berlin). 2019. Immobilienmarktbericht Berlin 2018/2019. Gutachterausschuss für Grundstückswerte in Berlin.

Sennett, Richard. 2018. Die offene Stadt. Eine Ethik des Bauens und Bewohnens. München: Hanser. 
Shrubsole, Guy. 2019. Who Owns England? London: William Collins.

Statistisches Bundesamt. 2020. Pressemitteilung Nr. 534 vom 28. Dezember 2020, https://www.destatis.de/DE/Presse/Pressemitteilungen/2020/12/PD20 534 61262.html, zugegriffen zuletzt am 11.1.2021.

Sutterlütti, Simon, und Stefan Meretz. 2018. Kapitalismus aufheben. Eine Einladung, über Utopie und Transformation neu nachzudenken. Hamburg: VSA Verlag.

Thurn, John Phillip. 2020. „Wer teil an der Gesellschaft hat. Grundeigentum, kommunales Vorkaufsrecht und Share Deal“. In Grundrechte-Report 2020, hg. von L.M. Armbruster et al., 132-135.

Tierney, Brian. 1997. The idea of natural rights, Studies on natural rights, natural law, and Church Law 1150-1625. Michigan: Scholars Press for Emory University.

Trautvetter, Christoph, und Markus Henn. 2020. Keine Transparenz trotz Transparenzregister. Ein Recherchebericht zu Anonymität im Berliner Immobilienmarkt, Studie im Auftrag der Rosa-Luxemburg-Stiftung, https://www.rosalux.de/filead$\mathrm{min} / \mathrm{rls}$ uploads/pdfs/Studien/Studien 5-20 Immobilien-Transparenz.pdf.

Vogel, Hans-Jochen. 1972. „Bodenrecht und Stadtentwicklung“. Neue Juristische Wochenschrift Nr. 35: 1544-1548.

Vogel, Hans-Jochen. 2018. „Bedarf des wirklich keiner Bodenrechtsreform? - Eine verdrängte Herausforderung“. Zeitschrift für Sozialökonomie Nr. 196/197: 1924.

Von Nell-Breuning, Oswald. 1983. „Bodeneigentum-Bodenpolitik-Bodenmarkt“. DASL-Berichte Nr. 8. Berlin.

Waltersbacher, Matthias. 2017. Bauland als Engpassfaktor für mehr bezahlbaren Wohnraum. Analyse der Baulandpreise aus den Kaufpreissammlungen der Gutachterausschüsse. Bonn.

Weigelt, Thomas. 2016. Die wachsende Stadt als Herausforderung für das Recht. Rechtliche Instrumente zum Erhalt und zur Schaffung heterogener Bevölkerungsstrukturen in der Innenstadt. Studien und Beiträge zum öffentlichen Recht. Bd. 26. Tübingen: Mohr Siebeck.

Weißmüller, Laura. 2018. „Der Boden gehört allen“. Süddeutsche Zeitung, 9.9.2018.

Wesche, Tilo. 2014. „Demokratie und ihr Eigentum. Von der Marktfreiheit zur Wirtschaftsdemokratie“. DZPhil 62(3): 443-486.

Wieland, Joachim. 2013. „Artikel 14“. In Grundgesetz Kommentar, herausgegeben von Horst Dreier und Hartmut Bauer, 1392-1499. 3. Auflage. Bd. 1. Tübingen: Mohr Siebeck.

Zentraler Immobilien Ausschuss (ZIA). 2019. „Mietendeckel: Enteignung durch die Hintertür wird Realität“. Pressemitteilung vom 30. Januar 2019, https://www. zia-deutschland.de/presse-aktuelles/presse-detail/news-single-pfad/mietendeckel-enteignung-durch-die-hintertuer-wird-realitaet/, zugegriffen zuletzt am 19.7.2020. 
